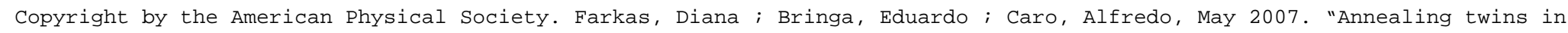
nanocrystalline fCC metals: A molecular dynamics simulation," PHYSICAL REVIEW B 75(18): 184111. DOI: 10.1103/

\title{
Annealing twins in nanocrystalline fcc metals: A molecular dynamics simulation
}

\author{
Diana Farkas, ${ }^{1}$ Eduardo Bringa, ${ }^{2}$ and Alfredo Caro ${ }^{2}$ \\ ${ }^{1}$ Department of Materials Science and Engineering, Virginia Tech, Blacksburg, Virginia 24060, USA \\ ${ }^{2}$ Chemistry, Materials and Life Sciences, Lawrence Livermore National Laboratory, Livermore, California 94550, USA
}

(Received 13 December 2006; published 23 May 2007)

\begin{abstract}
We report fully three-dimensional atomistic molecular dynamics studies of grain growth kinetics in nanocrystalline $\mathrm{Cu}$ of $5 \mathrm{~nm}$ average grain size. We observe the formation of annealing twins as part of the grain growth process. The grain size and energy evolution was monitored as a function of time for various temperatures, yielding an activation energy for the process. The atomistic mechanism of annealing twin formation from the moving boundaries is described.
\end{abstract}

DOI: $10.1103 /$ PhysRevB.75.184111

PACS number(s): 61.72.Mm, 61.82.Rx

Annealing twins in face-centered-cubic metals have been observed as early as $1897,{ }^{1-3}$ and are a prominent feature observed in routine metallography of these materials. The formation of these twins is usually associated with the process of grain growth that occurs during annealing at relatively high temperatures. Twin boundaries are usually flat and extend across an entire grain. The resulting density of twins after an annealing treatment is controlled by grainboundary energy, prior deformation, and resulting grain size. ${ }^{4-11}$ The formation of annealing twins is a very common experimental observation; the fraction of annealing twins typically increases with annealing time and therefore with the amount of grain growth. Up to two to three twins per grain are typically observed, and it has been suggested that the nucleation and growth of twinning are mechanistically linked to anomalous grain growth. ${ }^{12-14}$

In addition, it has been shown that imposed shear stresses during annealing of deformed fcc metals with low stacking fault energies tend to result in recrystallized microstructures containing higher fractions of twin boundaries than those annealed without an imposed shear stress. ${ }^{13}$ This appears to be true also for materials deformed by severe plastic deformation where high residual shear stresses are present.

The mechanisms by which annealing twins are formed are not completely understood. Current theories propose that accidents at growing grains and particularly faults on $\{111\}$ type planes are responsible for the formation of annealing twins. ${ }^{15-18}$ The models of twin formation generally require grain-boundary migration. In the model of Mahajan et al., ${ }^{15}$ the nucleation of Shockley partial dislocations at growth accidents on $\{111\}$ steps is associated with grain-boundary migration.

In nanocrystalline materials, there is strong evidence that deformation occurs through emission of Shockley partial dislocations from grain boundaries which are absorbed in the opposing grain boundaries. These mechanisms based on partial dislocations lead to twinning when partial dislocations are emitted in adjacent $\{111\}$-type planes. The deformation response of these materials has been studied using molecular dynamics simulation techniques, ${ }^{19-21}$ and similar theoretical work can help in the detailed understanding of the process of twin formation during annealing treatments.

In the present work, we report on relatively long molecular dynamics simulations of grain growth at zero external pressure and high temperature. We observe grain growth and twin formation. The nanometer size scale in the grains here is mandated by the viability of the molecular dynamics simulations. A large driving force for grain growth is present in the sample due to its nanoscale grain-boundary structure, driving the relatively fast grain growth observed in nanocrystalline materials. This, in turn, allows us to observe grain growth even in the short times accessible to molecular dynamics simulations. In the following, we describe our simulation technique and show results for the kinetics of grain growth and annealing twin formation. Finally, we discuss the observed mechanism of annealing twin formation at the atomistic scale.

Our sample is created with a Voronoi construction scheme and contains $\sim 300$ grains (approximately $1.6 \times 10^{6}$ atoms) with a mean grain size of $5 \mathrm{~nm}$. We used a $\mathrm{Cu}$ embedded atom method (EAM) potential developed by Sorensen et al., ${ }^{22}$ which predicts a stacking fault energy of $45 \mathrm{~mJ} / \mathrm{m}^{2}$. The simulation code used for the molecular dynamics simulations was LAMMPS. ${ }^{23}$

We first relaxed the as-created nanostructure at $T$ $=300 \mathrm{~K}$ and $p=0$ bar for $100 \mathrm{ps}$. This produced a stable microstructure for the study of the grain growth process. The
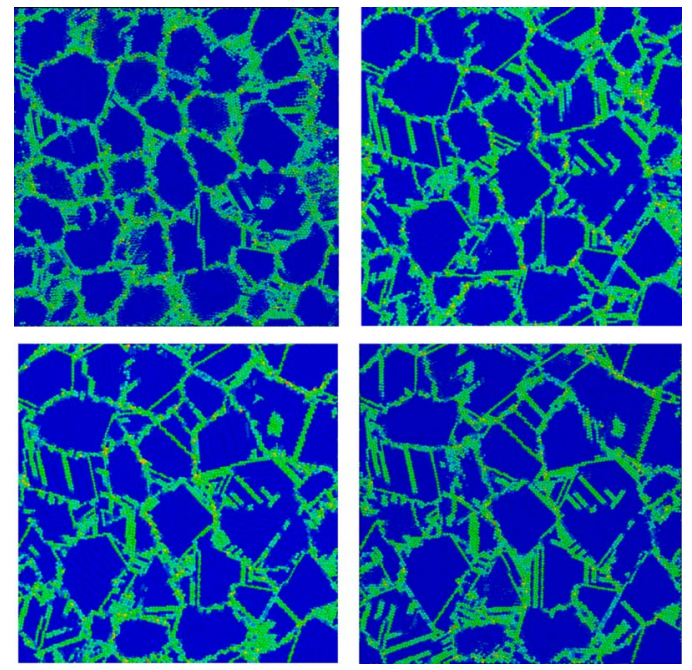

FIG. 1. (Color online) Time sequence for an annealing temperature of $800 \mathrm{~K}$. A representative sample slice is shown for annealing times of 25, 500, 1025 and 1500 ps. Color scale is given by the centrosymmetry parameter, with normal fcc atoms in blue. 

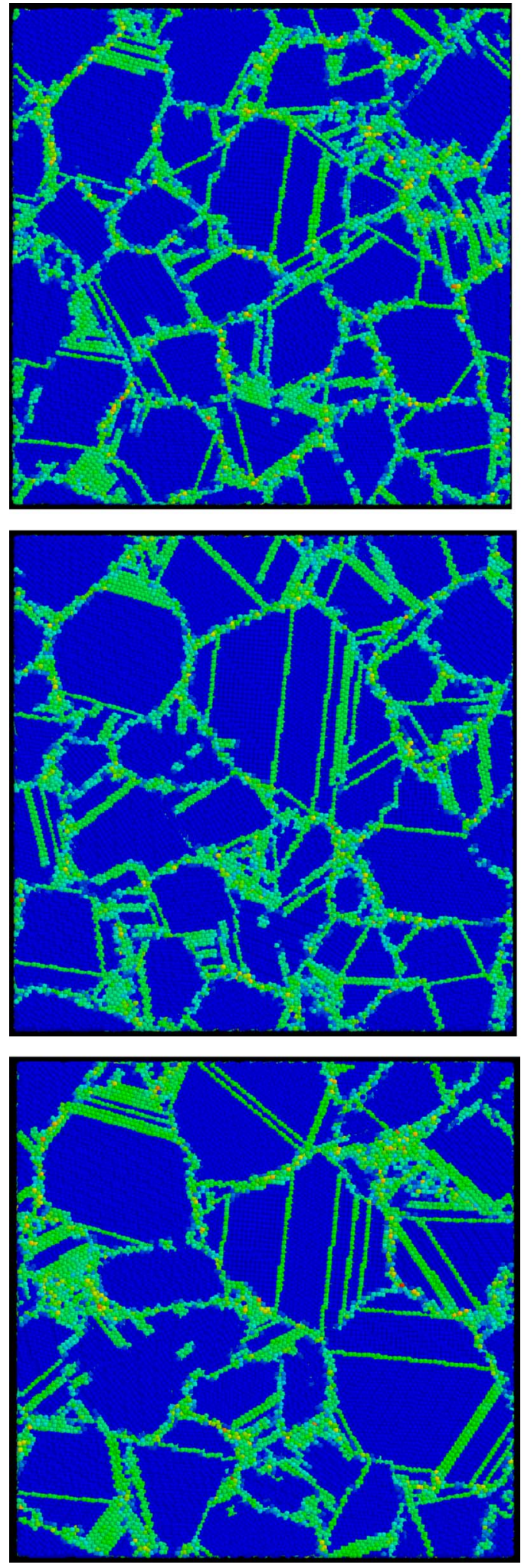

FIG. 2. (Color online) Temperature effect for an annealing time of $250 \mathrm{ps}$. A representative sample slice is shown for treatments at 800,1000 , and $1100 \mathrm{~K}$. Color scale is given by the centrosymmetry parameter, with normal fcc atoms in blue.

growth process itself was followed during annealing at various temperatures and zero pressure, as maintained by a
Nose-Hoover thermostat and barostat. As the treatment proceeds, snapshots of the sample were created for visualization and analysis of the grain growth process. The details of the induced grain growth were studied using visualization techniques based on the centrosymmetry parameter. ${ }^{24}$ We utilized massively parallel computational facilities to achieve long annealing times, by molecular dynamics standards, up to $1.5 \mathrm{~ns}$. This combination of techniques allowed the study of the kinetics of the grain growth process as well as the detailed mechanism of the formation of the observed annealing twins. Measurement of the grain size and annealing twin fractions was performed by standard counting techniques as the annealing process evolved the microstructure. In the following, we give the detailed results of the kinetics and mechanisms observed.

Figure 1 shows the time sequence evolution of the microstructure for an annealing temperature of $800 \mathrm{~K}$, with a representative sample slice shown after 25, 500, 1025, and 1500 ps. In this sequence, we can clearly observe grain growth and the appearance of annealing twins. Figure 2 shows the temperature effect for an annealing time of $250 \mathrm{ps}$ at 800,1000 , and $1100 \mathrm{~K}$. As expected, the higher the temperature, the more extensive these phenomena are. In order to quantify these results, the average grain size was obtained as a function of time using a simple standard technique of counting the number of grains in various sections of the sample to obtain a statistically meaningful average for the entire sample. The results of this procedure are shown in Fig. 3 for the treatment at $800 \mathrm{~K}$. In the initial part of the annealing treatment, linear grain growth was obtained. We note that in this process, the number of grains has decreased to about half the original number. The linear grain growth kinetics observed in the first 500 ps represents in itself a significant decrease in the total number of grains and is therefore not a transient regime, but rather a characteristic of the nanoscale sizes used here.

Both experimental and theoretical studies ${ }^{25-28}$ have pointed out that grain growth kinetics in nanocrystalline materials may be different from that in coarse-grained materials. The nanoscale effects in the kinetics can arise from a variety of factors, such as the role of triple junctions or grain rotation.

Continuing the analysis of the observed grain growth process, we followed the energy evolution of the samples as a function of time for various temperatures. The average potential energy per atom in the sample was monitored, and we observed that the energy per atom $E$ decreases as function of the time with a square root of time dependence as follows:

$$
E=E_{0}-s t^{1 / 2},
$$

where $E_{0}$ is the average (temperature-dependent) potential energy per atom in the initial equilibrated structure at $t=0$, and $s$ is a temperature-dependent constant. The values of $s$ and $E_{0}$ were obtained from this fitting analysis and are given in Table I, together with the $R^{2}$ values that indicate the accuracy of the linear fit. The values of $s$ can be plotted in Arrhenius form to obtain the activation energy for the process. Figure 4 shows these results, and the activation energy obtained for the grain growth process is $34.7 \mathrm{~kJ} / \mathrm{mol}$. These 


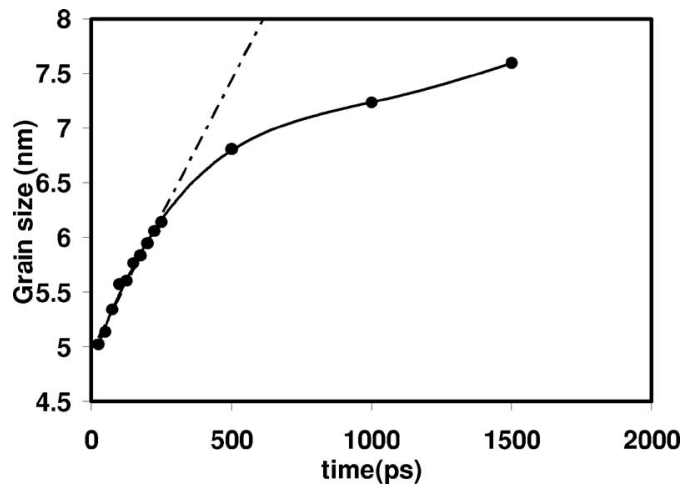

FIG. 3. Average grain size versus time obtained for the annealing at $800 \mathrm{~K}$.

values are of the order of magnitude expected for grainboundary self-diffusion in $\mathrm{Cu}$ and can be considered consistent with the migration kinetics being governed by the same individual atomic jump processes as grain-boundary selfdiffusion. Using the same interatomic potential, Suzuki and Mishin $^{29}$ found an activation energy of $53 \mathrm{~kJ} / \mathrm{mol}$ for the $\Sigma 5$ grain boundary in $\mathrm{Cu}$. Other grain-boundary (GB) mobility calculations, using different $\mathrm{Cu}$ potentials, have found similar values $(24-31 \mathrm{~kJ} / \mathrm{mol})$ for different types of GB. ${ }^{34-36}$

Figure 5(a) shows the number of annealing twins observed in an average cross section of the sample (the area is $716 \mathrm{~nm}^{2}$ ) as a function of time for the annealing treatment at $800 \mathrm{~K}$. The number of twins increases with time in a kinetics that closely resembles the kinetics of the observed grain growth. If the number of twins is divided by the number of grains in the same cross section of the sample, we can easily obtain an average number of twins per grain, which can be analyzed as a function of grain size. Figure 5(b) shows the number of twins per grain plotted as a function of grain size for the annealing treatment at $800 \mathrm{~K}$, indicating that the number of twins per grain varies linearly with grain size reaching values close to one twin per grain. At higher temperatures, more twins per grain are observed, as shown in Fig. 5(c). This figure shows the number of twins per grain observed after $250 \mathrm{ps}$ as a function of the annealing temperature.

The observed kinetics suggests that twins nucleate at grain boundaries during their migration at a more or less constant rate, and the number of twins produced is proportional to the distance of grain-boundary migration. This is in excellent agreement with the mechanisms proposed by $\mathrm{Ma}-$

TABLE I. Linear fitting results for the energy evolution as a function of the square root of time obtained at the various annealing temperatures. $E_{0}$ is given in $\mathrm{eV}$ and $s$ is in $\mathrm{eV} / \sqrt{\mathrm{ps}}$.

\begin{tabular}{lccc}
\hline \hline$T$ & $s$ & & $E_{0}$ \\
$(\mathrm{~K})$ & $(\mathrm{eV} / \sqrt{\mathrm{ps}})$ & $R^{2}$ & $(\mathrm{eV})$ \\
\hline 800 & $2.93 \times 10^{-4}$ & 0.95 & -3.286 \\
900 & $5.56 \times 10^{-4}$ & 0.89 & -3.254 \\
1000 & $7.815 \times 10^{-4}$ & 0.93 & -3.221 \\
1100 & $12.7 \times 10^{-4}$ & 0.90 & -3.184 \\
\hline \hline
\end{tabular}

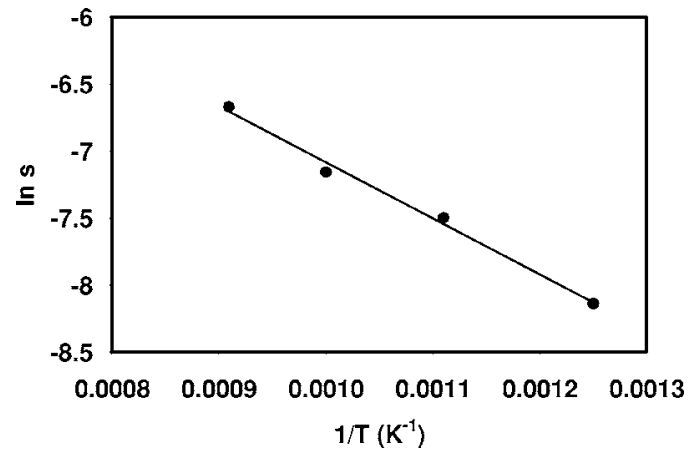

FIG. 4. Arrhenius plot of the energy evolution constant $s$ as a function of temperature.
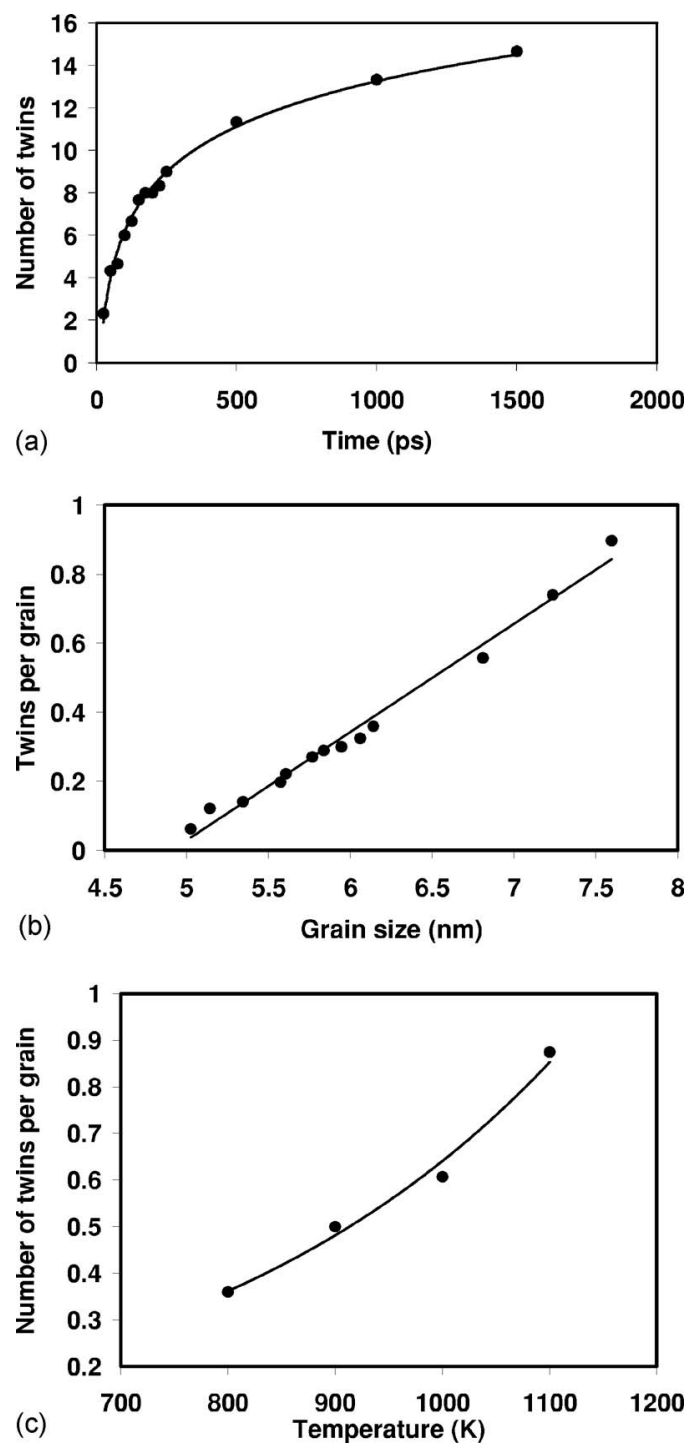

FIG. 5. (a) Number of twins observed in an average cross section of the sample as a function of time for the annealing at $800 \mathrm{~K}$. (b) Number of twins per grain observed as a function of grain size for the annealing at $800 \mathrm{~K}$. (c) Number of twins per grain observed after $250 \mathrm{ps}$ as a function of the annealing temperature. 

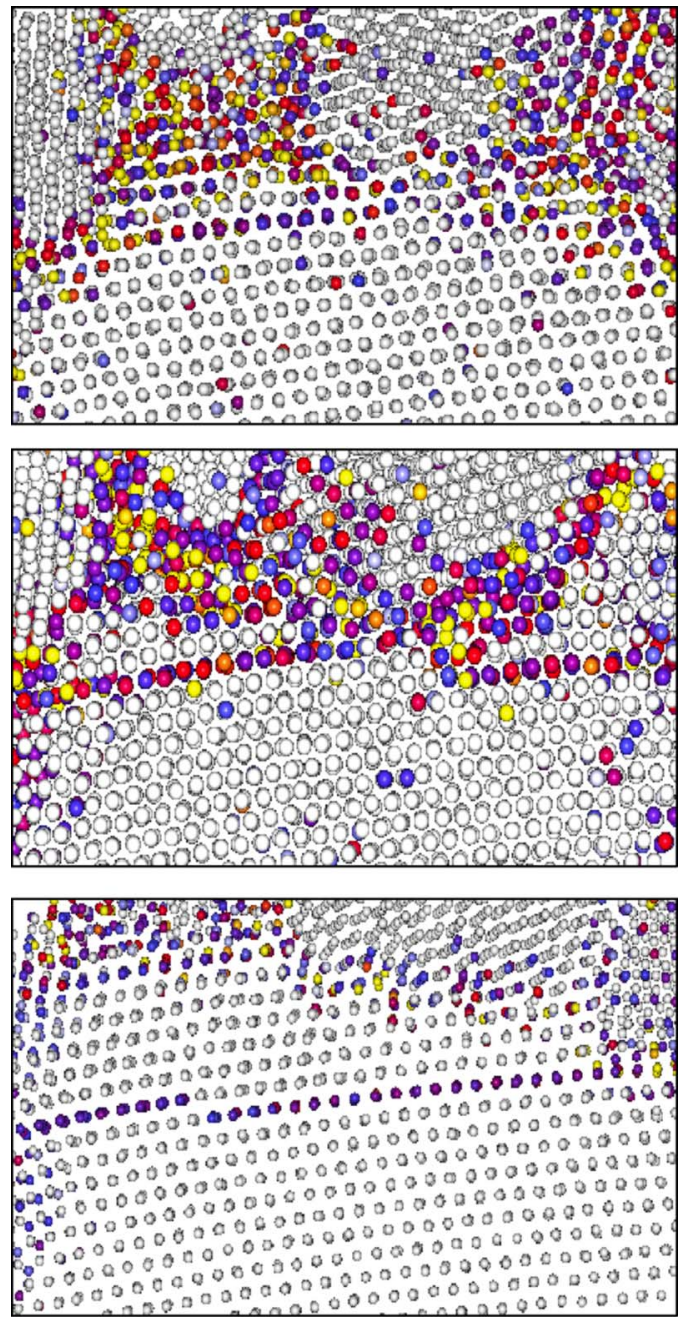

FIG. 6. (Color online) Sequence of events illustrating the mechanism of formation of the annealing twins. Color scale given by stress.

hajan et al. ${ }^{15}$ This model speculates that annealing twins in fcc metals and alloys form due to growth accidents on $\{111\}$ propagating steps present on migrating grain boundaries. As a result of the accidents, Shockley partials are generated at grain boundaries. These partials glide away from the boundary producing a twin. The model proposes that the formation of annealing twins entails the nucleation of Shockley partial loops on consecutive 111) planes by growth accidents on propagating [111] steps which are associated with a migrat- ing grain boundary. The model predicts that the higher the velocity of the boundary, the higher the probability of dislocation nucleation. Lateral growth of faults also occurs due to a repulsive interaction between Shockley partials constituting the twin boundary. Our simulations support the model where the twins nucleate and grow from the migrating boundaries through the emission of Shockley partial dislocations. The emission of the partials can be seen clearly in the stacking fault that the partials leave as they move. The sites of emission of the partials are mostly near triple points and near ledges, as suggested in previous models. An example of the observed mechanism is given in Fig. 6, which shows the sequence of events illustrating the mechanism of formation of the annealing twins. The first partial is nucleated near a triple point at the left, and additional partials nucleated in successive $\{111\}$ planes achieve the growth of the twin. In Fig. 6 this process is seen to occur from a point near the triple junction on the left first and then from a similar site located at the right. This process occurs simultaneously with the migration of the boundary upward.

As a summary, we have studied the formation of annealing twins in a nanomaterial. We find behavior consistent with experiments in both nanomaterials and micron-size polycrystals. Experimentally, it has been shown that heavily twinned nanomaterials offer exceptional mechanical and electrical properties. ${ }^{30}$ Twinning can result in additional hardening in nanowires ${ }^{31}$ and can be also produced in nanocrystals by ion implantation. ${ }^{32}$ Understanding how to control twin formation might lead to better engineered nanomaterials for various technological applications. We have found no particular reduction in the activation energy as seen in ceramic nanocrystalline materials. ${ }^{33}$ We have also found that in fcc nanocrystalline materials, twins appear in a mechanism that is similar to that postulated for larger grain materials, with an activation energy similar to that of grain-boundary diffusion. The activation energy that we found for grain-boundary migration of $34.7 \mathrm{~kJ} / \mathrm{mol}$ compares well with that found in previous studies in other fcc materials and with different interaction potentials which are in the the range of $24-31 \mathrm{~kJ} / \mathrm{mol}^{34-36}$

D.F. acknowledges support form NSF, Materials Theory Program, and the use of SYSTEM X, Virginia Tech's supercomputer. This work was performed under the auspices of the U.S. Department of Energy by University of California, Lawrence Livermore National Laboratory under Contract of No. W-7405-Eng-48.
${ }^{1}$ A. Liversidge, J. Chem. Soc. 71, 1125 (1897).

${ }^{2}$ J. A. Eving and W. Rosenhain, Philos. Trans. R. Soc. London, Ser. A 195, 279 (1900).

${ }^{3}$ H. C. H. Carpenter and S. Tamura, Proc. R. Soc. London, Ser. A 113, 161 (1926).

${ }^{4}$ R. L. Fullman and J. C. Fisher, J. Appl. Phys. 22, 1350 (1951).

${ }^{5}$ G. F. Boiling and W. C. Winegard, J. Inst. Met. 86, 492 (1957).

${ }^{6}$ C. Irving, A. P. Miodownik, and J. M. Tower, J. Inst. Met. 93,
360 (1964)

${ }^{7}$ J. M. Silcock, R. W. Rookes, and J. Barford, J. Iron Steel Inst., London 204, 623 (1966).

${ }^{8}$ W. D. Robertson and A. S. Tetelman, Strengthening Mechanisms in Solids (American Society of Metals, Metals Park, OH, 1962).

${ }^{9}$ W. Charnock and J. Nutting, Met. Sci. J. 1, 78 (1967).

${ }^{10} \mathrm{H}$. Hu and C. S. Smith, Acta Metall. 4, 638 (1956).

${ }^{11} \mathrm{M}$. A. Meyer and C. McCowan, in Interface Migration and Con- 
trol of Microstructure, edited by C. S. Pande, D. A. Smith, A. H. King, and J. Waller (American Society of Metals, Metals Park, $\mathrm{OH}, 1984)$, p. 125.

${ }^{12}$ N. Jia, Y. D. Wang, S. D. Wu, W. Z. Han, Y. N. Wang, J. N. Deng, and P. K. Liaw, Scr. Mater. 54, 1247 (2006).

${ }^{13}$ D. P. Field, R. C. Eames, and T. M. Lillo, Scr. Mater. 54, 983 (2006).

${ }^{14}$ S. L. Lee and N. L. Richards, Mater. Sci. Eng., A 405, 74 (2005).

${ }^{15}$ S. Mahajan, C. S. Pande, M. A. Imam, and B. B. Rath, Acta Mater. 45, 2633 (1997).

${ }^{16}$ R. L. Fullman and J. C. Fisher, J. Appl. Phys. 22, 1350 (1951).

${ }^{17}$ H. Gleiter, Acta Metall. 17, 1421 (1969).

${ }^{18}$ M. A. Meyers and L. E. Murr, Acta Metall. 26, 951 (1978).

${ }^{19}$ V. Yamakov, D. Wolf, S. R. Phillpot, and H. Gleiter, Acta Mater. 50, 5005 (2002).

${ }^{20}$ H. Van Swygenhoven, M. Spaczer, A. Caro, and D. Farkas, Phys. Rev. B 60, 22 (1999).

${ }^{21}$ H. Van Swygenhoven, A. Caro, and D. Farkas, Mater. Sci. Eng., A 309-310, 440 (2001).

${ }^{22}$ M. R. Sorensen, Y. Mishin, and A. F. Voter, Phys. Rev. B 62, 3658 (2000).

${ }^{23}$ S. J. Plimpton, J. Comput. Phys. 117, 1 (1995).

${ }^{24}$ C. L. Kelchner, S. J. Plimpton, and J. C. Hamilton, Phys. Rev. B
58, 11085 (1998).

${ }^{25}$ C. S. Pande and R. A. Masumura, Mater. Sci. Eng., A 409, 125 (2005).

${ }^{26}$ M. Hourai, P. Holdway, A. Cerezo, and G. D. W. Smith, Mater. Sci. Forum 386-388, 397 (2002).

${ }^{27}$ A. J. Haslam, S. R. Phillpot, D. Wolf, D. Moldovan, and H. Glaiter, Mater. Sci. Eng., A 318, 293 (2001).

${ }^{28}$ C. E. Krill III, L. Heflen, D. Michels, H. Natter, A. Fitch, O. Masson, and R. Birringer, Phys. Rev. Lett. 86, 842 (2001).

${ }^{29}$ A. Suzuki and Y. Mishin, Interface Sci. 11, 131 (2003).

${ }^{30}$ L. Lu, Y. F. Shen, X. H. Chen, L. Qian, and K. Lu, Science 304, 422 (2004).

${ }^{31}$ B. Wu, A. Heidelberg, J. J. Boland, J. E. Sader, X. M. Sun, and Y. D. Li, Nano Lett. 6, 468 (2006).

${ }^{32}$ Y. Q. Wang, R. Smirani, and G. G. Ross, Nano Lett. 4, 2041 (2004).

${ }^{33}$ S. Shukla, S. Seal, R. Vij, and S. Bandyopadhyay, Nano Lett. 3, 397 (2003).

${ }^{34}$ B. Schonfelder, D. Wolf, S. R. Phillpot, and M. Furtkamp, Interface Sci. 5, 245 (1997).

${ }^{35}$ H. Zhang, M. I. Mendelev, and D. J. Srolovitz, Acta Mater. 52, 2569 (2004).

${ }^{36}$ S. M. Folies and J. J. Hoyt, Acta Mater. 54, 3351 (2006). 